\title{
Role of Center of Excellence in fostering Health based Research in Africa
}

\author{
Mohamed L. Salem \\ Prof. of Immunology, Faculty of Science \\ Director, Center of Excellence in Cancer Research, Tanta University, Egypt \\ Editor-in-Chief, J Cancer and Biomedical Research
}

\section{IIII}

ABSTRACT

Recently, academicians and clinicians have been focusing their researches on solving scientific challenges meetings the medical sciences using technologies developed in the last decades. This research has led to a growing body of scientific data in the field of biomedical research that led to breakthrough discoveries in health and disease in particular cancer. These discoveries are based on inter-, multi-, and trans-disciplinary approaches. Although both multi- and inter-disciplinary research in the last decades have led to major findings with paramount significance in translational research from bench to clinic, the coming era requires more trans-disciplinary research based on sophisticated networking between researchers from different disciplines. Ultimately, multidisciplinary Research draws on knowledge from different disciplines but stays within their boundaries. Interdisciplinary research analyzes, synthesizes and harmonizes links between disciplines into a coordinated and coherent whole. Trans-disciplinary research integrates the natural, social and health sciences in a humanities context, and transcends their traditional boundaries. One approach to accomplish the generation and maintenance of trans-disciplinary research is through founding center of excellence in research which should have the core facilities required for such aim.

In African countries, few center of excellence in research cover the gap between basic biological sciences and translational biomedical sciences. As such, we believe that the already founded centers as well as founding new canters could be a new platform to cover this gap. These centers should focus on fostering such gap through focusing on interdisciplinary studies. By having these centers, I would expect emergence of publication of several studies with paramount significance to the advancement of cutting-edge research based on basic and recent technologies including single cell analysis, proteomics and genomic arrays. Definitely, making a network of these centers in Africa is a must for strengthen the scientific collaboration in health based research which ultimately will aid patients, physicians and scientific community.

\section{II ARTICLE INFO}

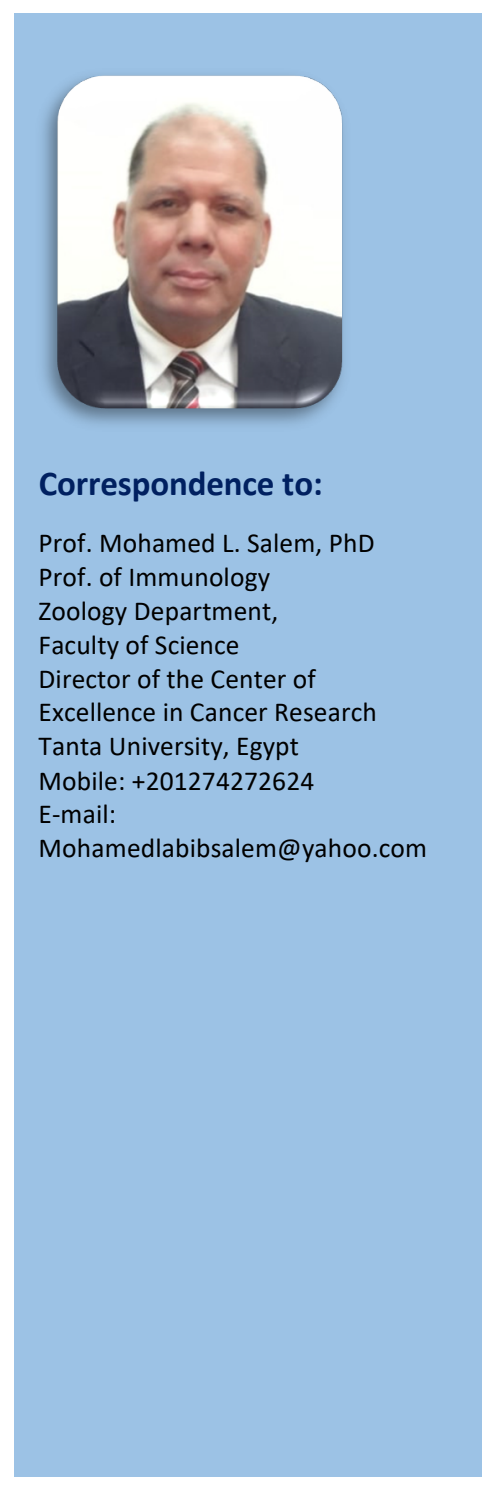

